\title{
ANATOMY AND PHYSIOLOGY OF SUPERNUMERARY CERCAL AFFERENTS IN CRICKETS: IMPLICATIONS FOR PATTERN FORMATION $^{1}$
}

\author{
R. K. MURPHEY, S. E. JOHNSON, AND D. S. SAKAGUCHI
}

Neurobiology Research Center, Department of Biological Sciences, State University of New York at Albany, Albany, New

York 12222

Received June 1, 1982; Revised August 20, 1982; Accepted August 24, 1982

\begin{abstract}
The afferent projection of supernumerary sensory neurons was examined in the cricket cercal sensory system. When a right cercus was exchanged for a left cercus the growth of supernumerary cerci was induced. The supernumerary cerci which formed tended to appear medial and/or lateral in a simple left $\rightarrow$ right exchange and were always right handed. Distal to the region where transplant and supcrnumerary meet, receptors on the supernumerary structure appear in a recognizable pattern typical of control cerci, and individual receptors homologous in position to control receptors could be identified. The supernumerary neurons associated with these identified receptors projected to the same area of neuropil as their homologs and thus uniquely identified neurons by all of the usual criteria were produced by the regeneration process. When the response properties of one postsynaptic neuron were examined it was shown that supernumerary afferents innervated it in a normal manner.

The supernumerary neurons provide tests of various hypotheses for the assembly of ordered afferent projections. First, their axon trajectories are often atypical, but the terminal arbors grow into their proper areas in spite of this. Thus axon trajectory can be uncoupled from target area and is therefore unlikely to be a decisive factor in growth of the arbor. Second, supernumerary neurons are born later in development than their control homologs but their aroborizations are completely normal, adding to the existing evidence against a timing mechanism in the assembly of this afferent projection. Third, supernumerary neurons share target regions with their homologs, indicating a lack of competitive interactions. The most economical hypothesis to explain the precise mapping between the cercus and CNS holds that one step in the differentiation of insect sensory neurons is position dependent and that this step determines the choice of a target region within the CNS.
\end{abstract}

A very important step in the development of all nervous systems is the connection of the sensory systems with the rest of the central nervous system (CNS). This critical step in nervous system development has been the object of much study, the result of which is the indisputable fact that many sensory systems are organized somatotopically. The mechanism by which the highly ordered growth of afferent neurons produces a topographically correct map of the periphery remains obscure and numerous hypotheses have been advanced to explain it.

\footnotetext{
' W. Walthall, G. Jacobs, and Dr. J. P. Bacon provided technical and editorial assistance throughout the course of this work. Dr. John Schmidt provided insightful comments and suggestions concerning the analysis of competitive interactions. L. Welch, R. Loos, and R. Speck provided endless help during manuscript preparation. This work wus supported by National Science Foundation Grant BNS782493901 and National Institutes of Health Grant NS15571A.

${ }^{2}$ To whom correspondence should be addressed.
}

The pendulum has swung from the purely mechanical guidance hypothesis of Weiss (Weiss and Taylor, 1944) to the very influential chemoaffinity model of Sperry (1963) and is presently suspended somewhere in between. The process of assembling a somatotopic map is seen in an extreme form among the insects and other arthropods. In these animals the epidermally derived sensory neurons are generated independently in time and space (Lawrence, 1966) of the CNS (Bate, 1976) and yet grow to the CNS and synapse there in an orderly manner (Murphey, 1981). This peripheral location of sensory neurons, combined with the relative ease with which insect epidermis can be transplanted and the vigorous regeneration of sensory cells after transplantation makes insects attractive for studies designed to understand the mechanisms which underlie the assembly of ordered sensory projections.

The epidermal origin of insect sensory neurons and the fact that their terminal arborizations in the CNS are 
correlated with their position on the body surface suggest that their location at the time of origin in the epidermis may influence or determine their pattern of arborization (Bate, 1978; Palka, 1980). Thus the epidermal origin of insect sensory neurons focuses attention on pattern formation in the epidermis itself, about which a good deal is known. Most analyses of pattern formation in the insect epidermis are dependent on a model made explicit by Wolpert (1969). The heart of this model is the hypothesis that cells are in some way informed of their position in an array of cells and that this "positional information" can direct the differentiation of cells in the array. The model was developed to help interpret the results of epidermal transplantation experiments which induced regeneration near the borders of the grafted tissues. In insects the transplantation of limbs to ectopic sites, such as from the right to the left side of the body, often leads to the production of supernumerary limbs. Modifications of Wolpert's (1969) positional information model predict the location and symmetry of such supernumerary structures and the accuracy of such predictions is considered to be a test of the model (French et al., 1976).

Since supernumerary limbs are a central feature of developmental studies and are one form of regeneration which we suggest might support the positional information model, we have induced the production of supernumerary limbs and examined the anatomy and synaptic connectivity of sensory neurons on such appendages. Supernumerary cerci were induced by exchanging a right for a left cercus (Palka and Schubiger, 1975). We demonstrate first that the external structure of supernumerary cerci obeys all of the rules of symmetry established by developmental biologists for regenerating insect limbs and our results extend this conclusion to the level of identified cells. Second, we show that supernumerary neurons produced by the regeneration process project to the CNS in accord with their location on the supernumerary structure. The central terminations of these supernumerary neurons, their axon trajectories, and the timing of their growth have important implications for the various models of pattern formation in the CNS and these are considered in the discussion.

\section{Matcrials and Methods}

Two types of surgery were performed to produce supernumerary cerci; right cerci were replaced with left cerci, maintaining the proper dorsoventral polarity $(N$ $=259$ ), and right cerci were replaced with left cerci, after inverting the dorsoventral axis $(N=254)$. Most surgeries were performed in the sixth instar $(N=329)$; the remainder were performed in the fifth $(N=122)$ and seventh instars $(N=62)$. In all cases cerci were transplanted from one specimen to another of the same age. Of these specimens 27\% (142 of 513) were useful for further analysis as indicated by the existence of at least one supernumerary cercus with a relatively normal array of clavate hairs. Half of these (71 of 142) had two useful supernumerary cerci. As a control procedure, we also prepared 55 "regenerate" cerci by removing the cercus in the sixth instar and allowing the specimen to regenerate a cercus.
Our staining methods have been published in detail elsewhere (Murphey, 1981). The method successfully stained single neurons in $68 \%$ of our attempts in the present series of experiments. Table I provides a breakdown of the neurons stained.

Electrophysiological procedures have been described in detail elsewhere (D. S. Sakaguchi and R. K. Murphey, manuscript in preparation). Briefly, extracellular recordings were obtained from the abdominal connectives while the animal was displaced on a goniometer stage. Positionsensitive neurons were identified and their action potential frequencies were determined during static displacements. The responses were plotted in polar coordinates.

\section{Results}

The occurrence and structure of supernumerary cerci. When a right cercus was amputated and replaced by the left cercus of a sister specimen, supernumerary cerci were produced. The supernumeraries formed at the host/graft junction where the match between host and graft tissue was poorest. For example, when the exchange maintained the dorsal-ventral orientation of the cercus, there was a mismatch medially and laterally; the lateral aspect of the transplanted cercus was apposed to the medial aspect of the socket and thus a supernumerary cercus would form medial to the transplant (see Fig. 1A). Similarly, if the left cercus was rotated $\left(180^{\circ}\right)$ about its long axis before being inserted in the right socket, the dorsal and ventral areas of host and graft were mismatched and supernumeraries appeared in these areas. The supernumerary cerci produced in both operations were right cerci. Thus, in many cases an animal possessed three cerci on the

TABLE I

Neurons stained

\begin{tabular}{lc}
\multicolumn{2}{c}{ Neurons stained } \\
\hline Neurons & Number of Successful Injections \\
\hline Supernumerary & 18 \\
10 & 13 \\
$11-19$ & 11 \\
40 & 19 \\
60 & 3 \\
80 & 5 \\
Miscellaneous & \\
Regenerate & 5 \\
10 & 10 \\
40 & 14 \\
60 & 3 \\
80 & \\
Transplant & 8 \\
10 & 11 \\
20 & 23 \\
40 & 38 \\
60 & 8 \\
80 & 4 \\
90 & \\
Control & \\
10 & 28 \\
$11-19$ & 16 \\
20 & 12 \\
40 & 57 \\
60 & 82 \\
80 & 18 \\
90 & 11 \\
&
\end{tabular}



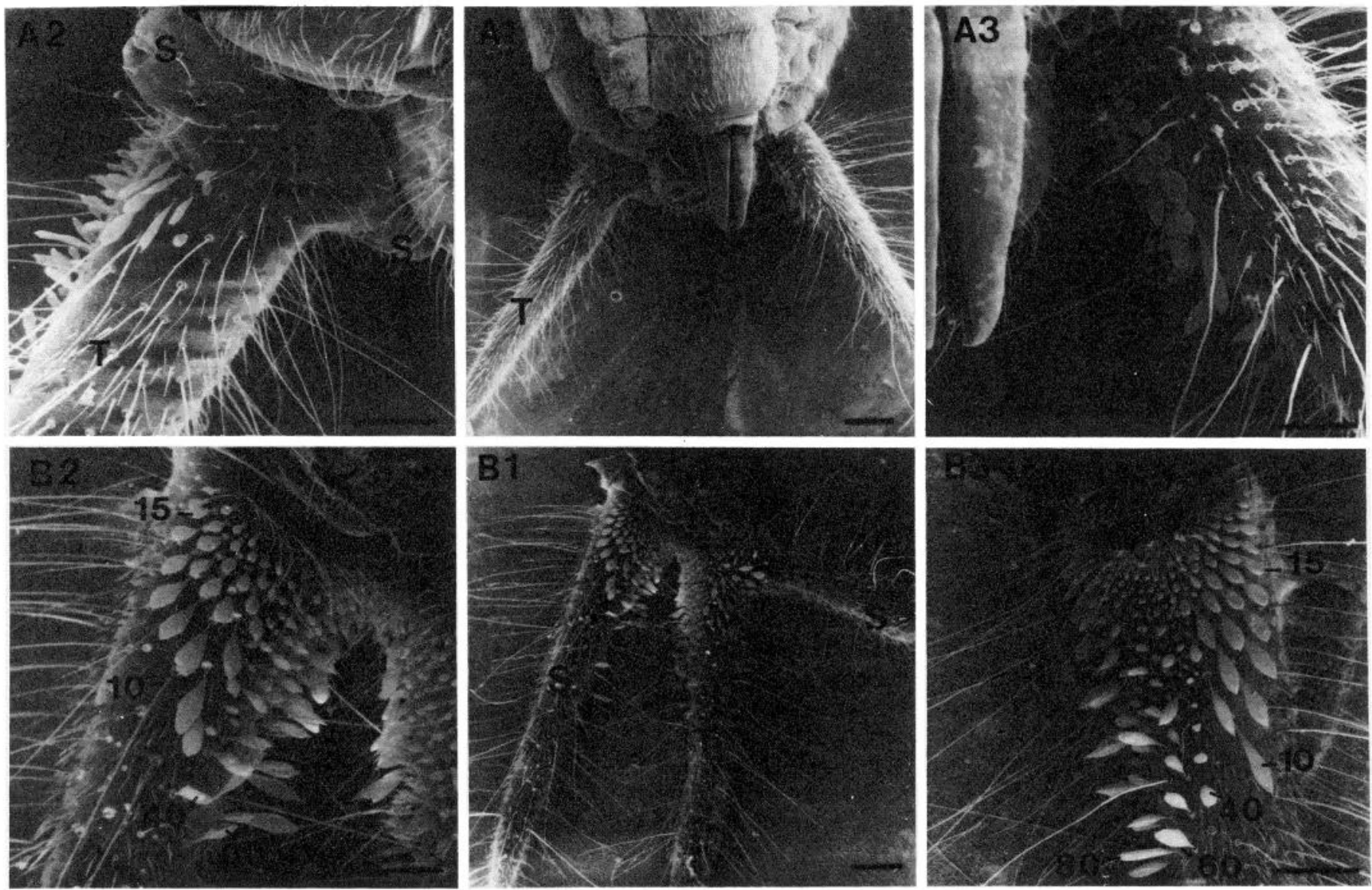

Figure 1. The structure of supernumerary cerci. A, The structure of supernumerary cerci on a seventh instar specimen (the transplantation occurred in the fifth instar). Note the lack of clavate hairs on the supernumerary buds. A1, Low magnification photograph of the entire genital region. $A 2$, Detail of the supernumerary buds. $A 3$, Detail of the proximal region of the control cercus. Calibration bars: $A 1,600 \mu \mathrm{m} ; A 2$, and $A 3,200 \mu \mathrm{m}$. B, The structure of cerci from adult specimens. $B 1$, Low magnification view of a transplanted cercus with its two supernumeraries. $B 2$, Detail of ventral hairs on a supernumerary cercus. Note the similarity of the orientation of the hairs and rows on the supernumerary and control cerci. B3, Detail of the ventral clavate hairs on a control cercus. The receptors discussed in the text are labeled. $S$, supernumerary cerci; $T$, transplanted cercus. Calibration bars: $B 1,600 \mu \mathrm{m} ; B 2$ and $B 3,200 \mu \mathrm{m}$.

right side: two right-handed supernumeraries and one left-handed transplant. The handedness of each cercus was easy to discern because the cone-shaped cercus possesses a peculiar group of club-shaped receptors, called clavate hairs, only on the medial aspect of the cercus. These results confirm those of Palka and Schubiger (1975), who had previously described the gross structure and symmetry of such supernumeraries using the clavate hairs as a landmark, and are consistent with previous work on cockroach legs (French et al., 1976).

The transplantation was carried out in the fifth, sixth, or seventh instar. After a single molt a supernumerary bud may appear but it never had clavate hairs on it. After a second molt (19 days postoperative) the first individuals processing clavate hairs were detected but most possessed no clavate hairs at all (Fig. 1A). Beginning three molts after the operation (24 days postoperative) many identifiable clavate receptors were present. Thus the earliest any individual possessed clavate receptors was 19 days and the earliest we could uniquely identify a given receptor was 24 days postoperative.

The clavate hairs on a control cercus are arrayed in rows running nearly parallel to the long axis of the cercus and hairs in different rows have different orientations with respect to the cercal surface (Fig. 1B3). Normally, hairs in row 10 are tilted away from the body, hairs in row 40 are normal to the cercal surface, hairs in row 60 are tilted toward the body, and those in row 80 are again normal to the surface. This orderly structure makes it possible to identify homologous receptors on different cerci (Murphey, 1981).

We were able to identify many of the same receptor rows as well as individual receptors in each row on supernumerary cerci (Fig. 1B2). Since each receptor is formed by an invariant clone of four cells (Lawrence, 1966), one of which is the sensory neuron, regeneration produced a supernumerary array of neurons which is accurate at the single cell level. This accuracy was demonstrated for distal neurons in the clavate array. Near the base of the structure, where supernumerary and transplant meet, there were many sensory receptors which had no corresponding structure on a normal cercus (Fig. 1B1).

Afferent projection from supernumerary cerci. The 

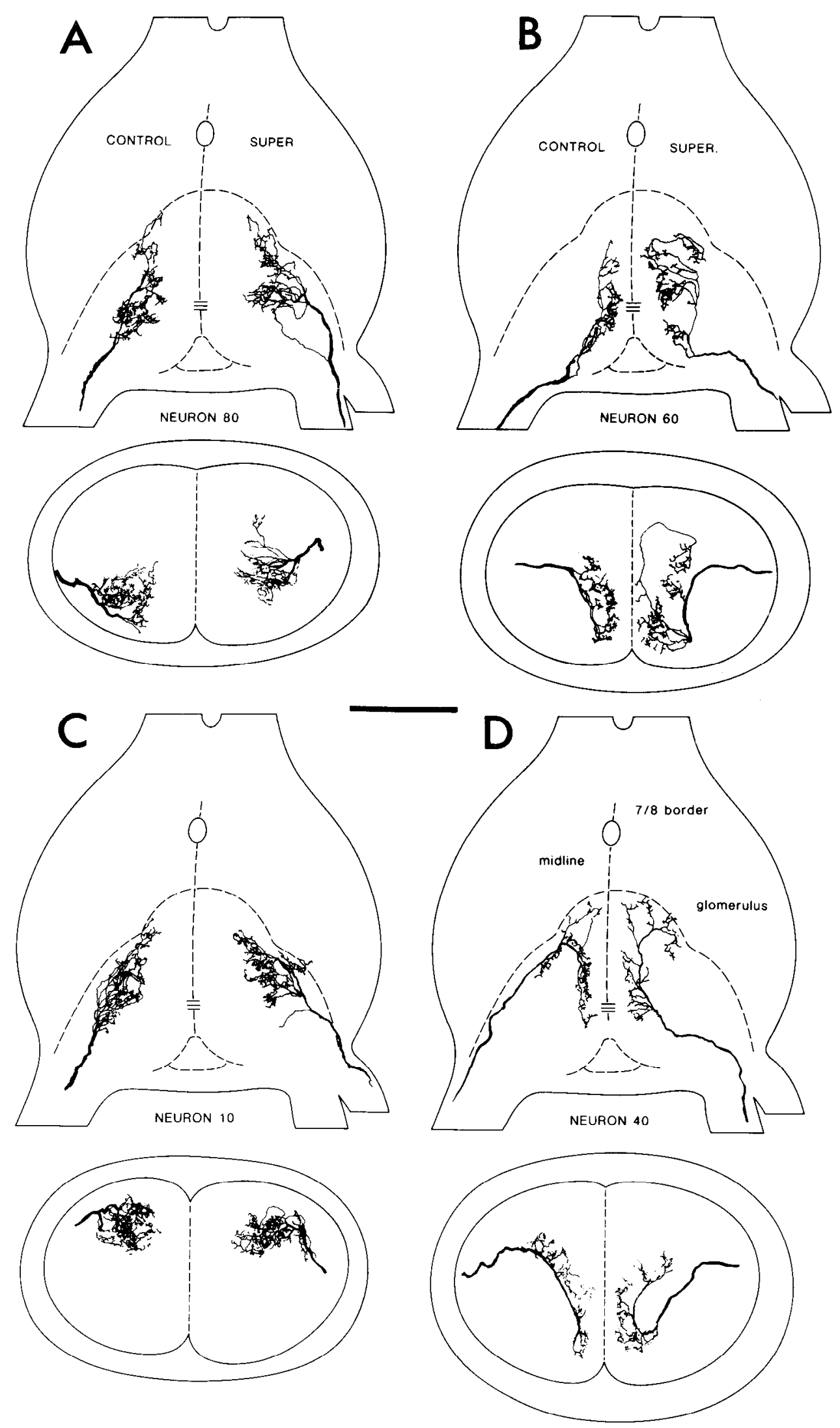

Figure 2. The terminal arborizations of clavate sensory neurons located at four positions within the clavate array. The receptors which were stained, numbers 10,40,60, and 80, are indicated in Figure 1, B2 and B3. The left side of each figure is a camera lucida drawing of a control neuron; the right side is a drawing of the homologous neuron on the supernumerary cercus of the same specimen. The arbors were aligned on the standard outline by referring to internal landmarks visible in whole mount including the midline, commissural process of giant neurons, and the area of neuropil at the rear of the ganglion representing embryonic segment 11. The circle on the midline represents the hole through the ganglion between the embryonic 7 th and 8th ganglion. The upper panels are dorsal views, the lower panels are cross-sectioned views. The approximate extent of the cercal glomerulus is indicated by the dashed line and is included to aid the reader. While the glomerulus is visible in ethyl gallate sections, it is not detectable in whole mounts and this outline is only an approximation. Calibration bar, $100 \mu \mathrm{m}$. 


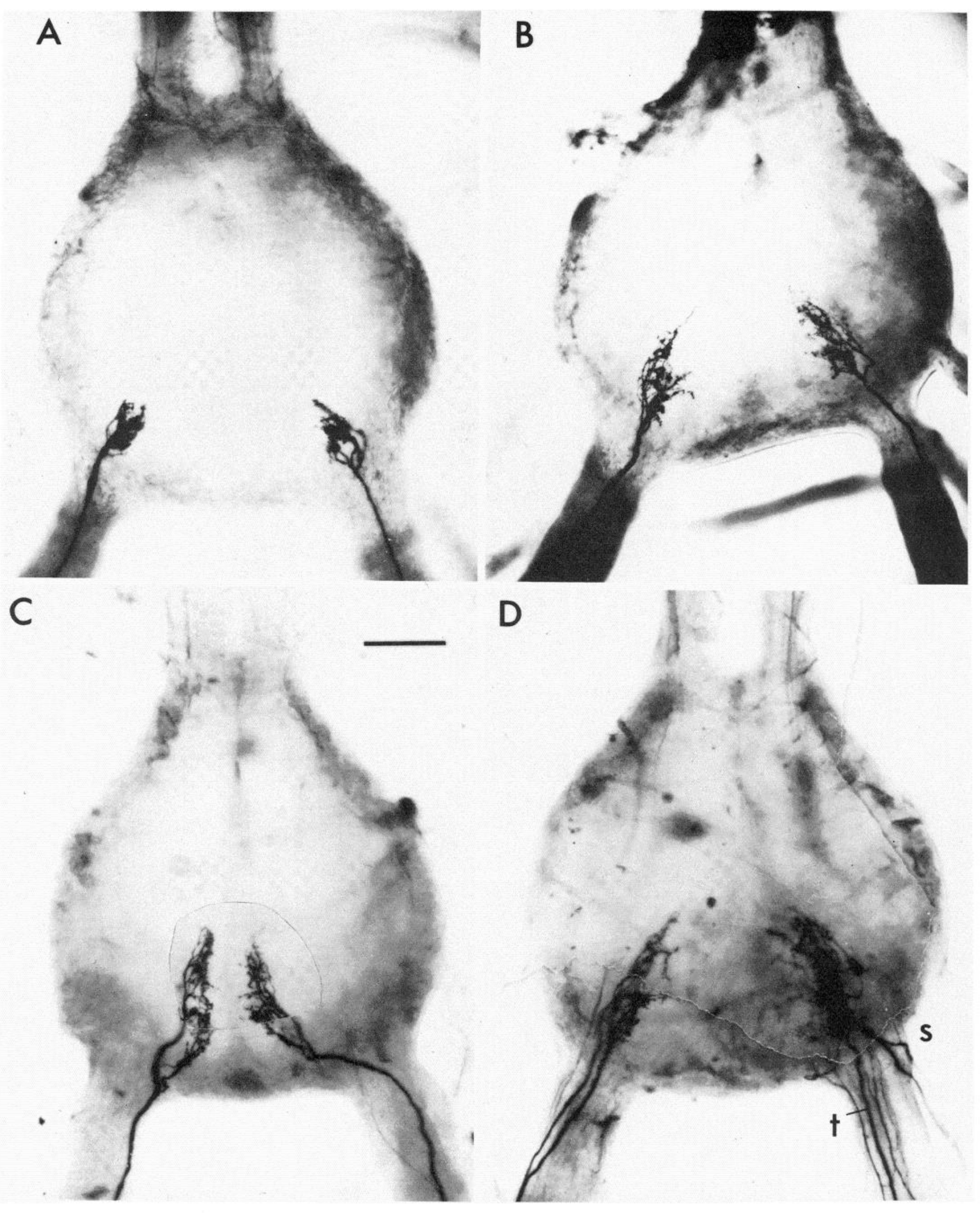

Figure 3. Photographs of sample preparations. Experimental neurons are on the right and controls are on the left. A, A specimen in which right supernumerary neuron 15 and left control neuron 15 were stained. $B$, A specimen in which a right regenerate neuron 10 and a left control neuron 10 were stained. $C$, A preparation in which right supernumerary neuron 60 and left control neuron 60 were stained. $D$, A specimen in which neurons 10 and 11 on a transplant cercus (right) and neuron 10 on a supernumerary cercus as well as neurons 10 and 11 on the control cercus (left) were stained. Note the overlapping arborizations of supernumerary and transplanted neurons on the right side of the ganglion. $S$, supernumerary cerci; $t$, transplanted cercus. Calibration bar, $100 \mu \mathrm{m}$. 
central question which motivated the present work was, where do supernumerary neurons arborize in the CNS? The region where the sensory neurons arborize and synapse has been called the cercal glomerulus (Edwards and Palka, 1974; Shankland, 1981). It is a hollow club-shaped region of neuropil located in the terminal abdominal ganglia of crickets and other orthoptera. This glomerulus stains differentially with respect to the surrounding neuropil (Shankland, 1981; Jacobs, unpublished observation) and is delineated by dashed lines in Figures 2 and 5 . As the axons of mechanosensory neurons approach the glomerulus, they enter two main tracts: one dorsolateral and one ventromedial (Shankland, 1981; Murphey, 1981). The axons of neurons in rows 10 to 50 project along the dorsolateral pathway and axons from rows 60 to 90 follow the ventromedial tract. In general, a sensory neuron's soma position on the cercus is correlated with the location of its arbor position in the glomerulus and near neighbors arborize near one another. Just as each neuron can be identified by the row to which it belongs and its position within the row, the location of the arbors can be described by their general position around the circumference of the glomerulus and their anterior/posterior extent within that region (Murphey, 1981).

In our analysis we were concerned with whether supernumerary neurons arborized in a manner similar to that of homologous neurons on a control cercus. We first examined the area of arborization of neurons 10, 40,60, and 80 , which provide a sample of neurons whose location would normally outline the glomerulus. As Figures 2 and 3 demonstrate, the supernumerary neurons arborize in areas which correspond to those of the homologous neuron on the control cercus. While there was some variability in axon trajectory (see below) and the details of the arborization, it was clear that distal neurons throughout the clavate sensory array map in essentially the same manner as neurons at homologous positions on the control cercus (summarized in Fig. 4).

In order to establish the correspondence between proximodistal position within a row and arbor position within the glomerulus, we examined neurons in row 10 where this correlation is most firmly established (Murphey et al., 1980; Murphey, 1981). As shown in Figures $3 A$ and 5, a proximal neuron such as neuron 15 arborized more posteriorly in the ganglion than neuron 10 , just as in the control nervous system. Thus the data of Figures 2 and 5 demonstrate that the afferent projection between the periphery and the center is normal for supernumerary cerci.

Superimposed afferent projections. Neurons of the transplanted cercus, the one which was used to induce supernumerary growth, projected to the ganglion normally. This is shown for neurons $10,40,60$, and 80 in Figure 6. Clearly, the neurons on the transplanted left cercus arborized in the right glomerulus appropriate to their position on the cercus. The left neuron 10 , for example, when transplanted to the right side of the animal, arborized in the area of the right glomerulus appropriate for right neuron 10 (Fig. 6C).

This implies that supernumerary neurons and their transplanted homologs are projecting to the same target areas. In fact, as many as three homologs may exist in these experiments (one transplant and two supernumer aries). We examined the terminal arborizations of pairs (one transplant, one supernumerary) or triplets (one transplant, two supernumeraries) of such cells to determine how the afferent projections were organized spatially with respect to one another. It was clear that two
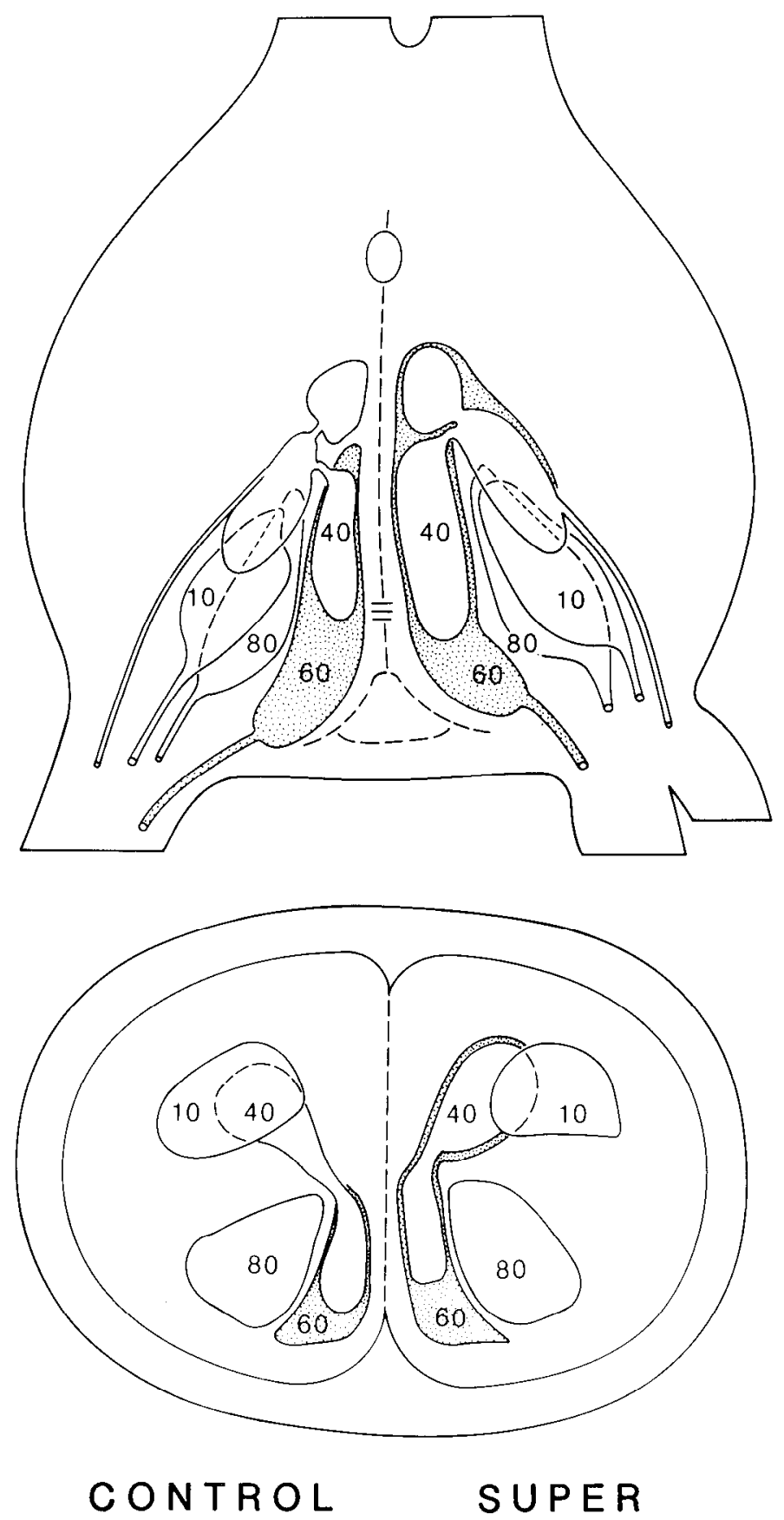

Figure 4. A summary of the projection areas for four neurons on supernumerary and control cerci. The outlined areas were obtained by aligning all of the neurons of a given identity on the standardized outline and then outlining the projection area. Thus, the outline for supernumerary neuron 60 would encompass the terminal arborizations of all examples of this cell. Note the similarity between the control and supernumerary projections. The differences are mainly a broadening of the domain for a given cell which is most obvious for cell 60 but true for all cells. 
neurons could occupy the same target area by interdigitating (Figs. $3 D$ and 7). There was no evidence that the transplanted and supernumerary afferent projections divided the target into separate, cercus-specific domains.

Another possible consequence of these duplicate projections was the shrinkage of the terminal arbors of each cell as a result of some competitive interaction (Murray et al., 1982). In order to assess this possibility, we examined the terminal arborizations of neuron 60 quantitatively. We took as our quantitative measure the number of varicosities in the terminal arborization (Fig. 8). Many of these varicosities are presynaptic structures (Straus-
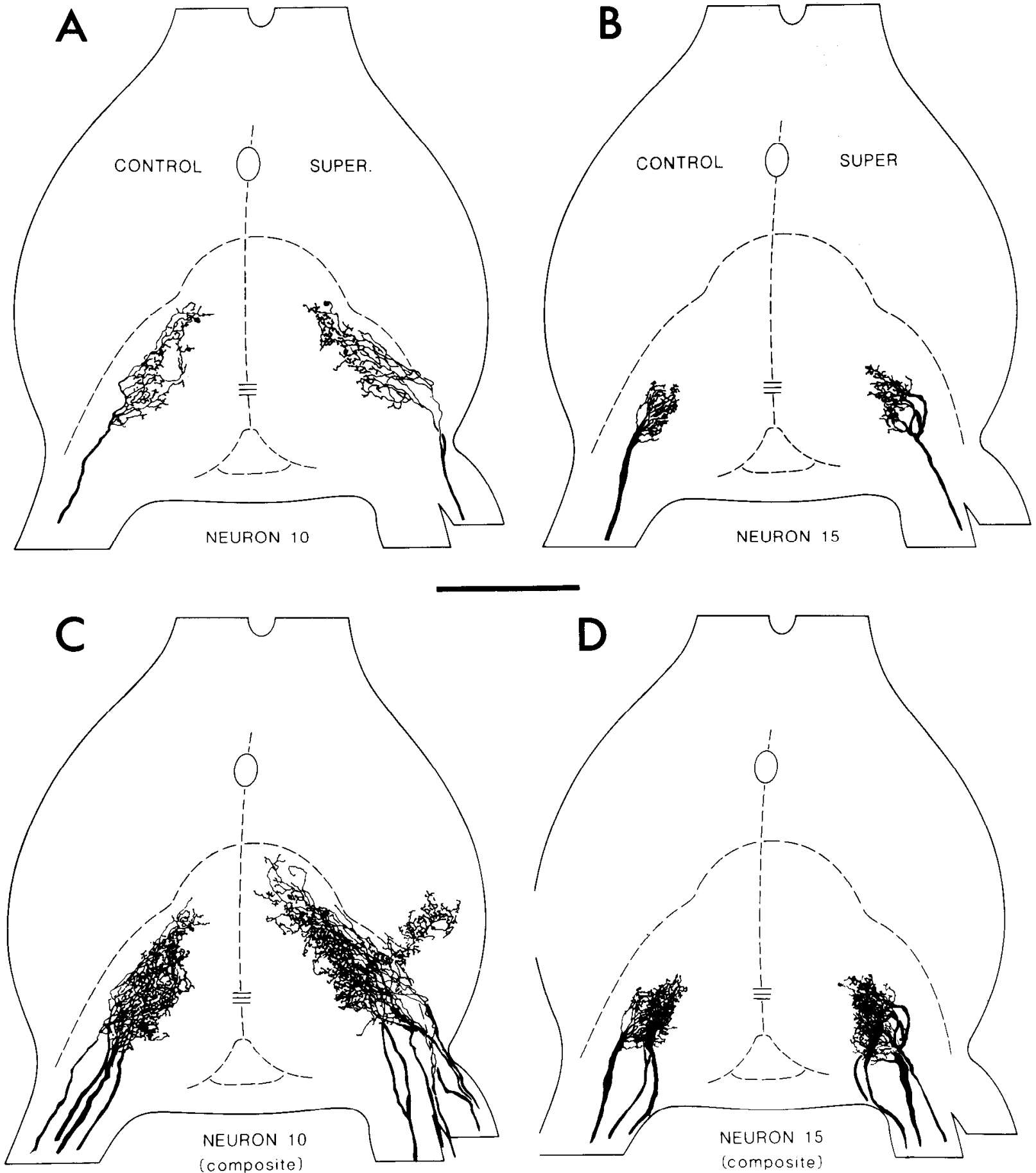

Figure 5. Differences in the arbors of neurons within a row of receptors. $A$, Control and supernumerary neuron $10 . B$, Control and supernumerary neuron 15. $C$, Composite of all of the supernumerary neuron $10 \mathrm{~s}$ and their corresponding controls standardized for ganglion size and drawn on a single ganglion outline. Note that only one supernumerary neuron 10 arborized outside the glomerulus. $D$, Composite of all supernumerary $15 \mathrm{~s}$ and their controls drawn on a single standardized ganglion. Note that none of these neurons invade the anterior region of the glomerulus. Calibration, $100 \mu \mathrm{m}$. 

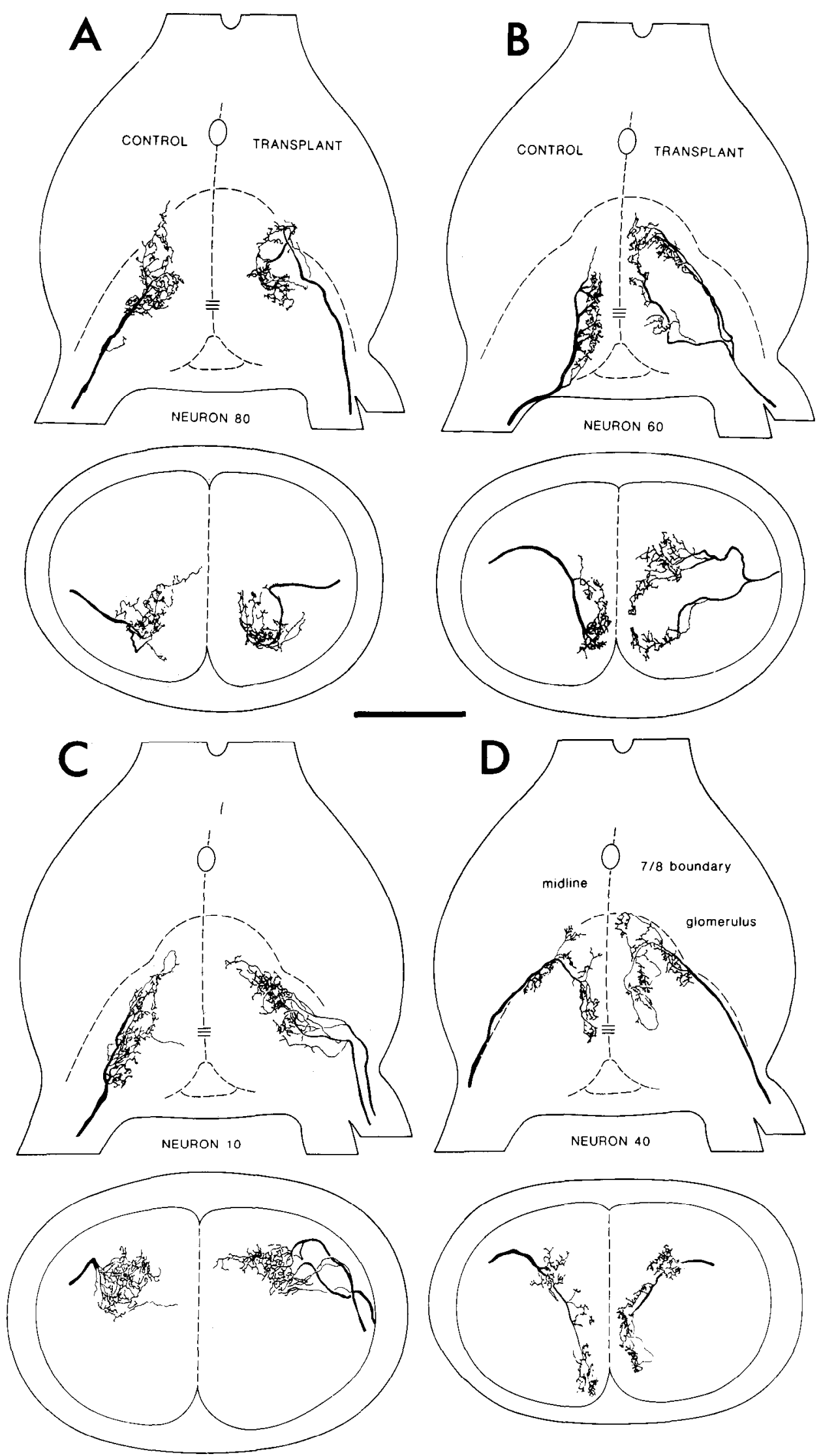

Figure 6. The terminal arborizations of clavate neurons located on the transplanted cercus. In $A, B$, and $C$, the control and transplant neuron were stained in the same specimen; the two neurons in $D$ come from separate specimens. 
feld, 1971; Muller and McMahan, 1976; DeRiemer and Macagno, 1981) but we cannot say whether all varicosities are synapses. Thus the varicosities were simply counted as one measure of the complexity of the terminal arbor. The total number of varicosities for normal neurons was relatively invariant $(226 \pm 20)$ and this encouraged us to compare various experimental neurons (Table II). Specifically, we compared regenerate and supernumerary neurons because these were produced at the same time in the life of the animal, but regenerate neurons have no homologs to interact with, thus serving as an appropriate control. Our assumption was that these

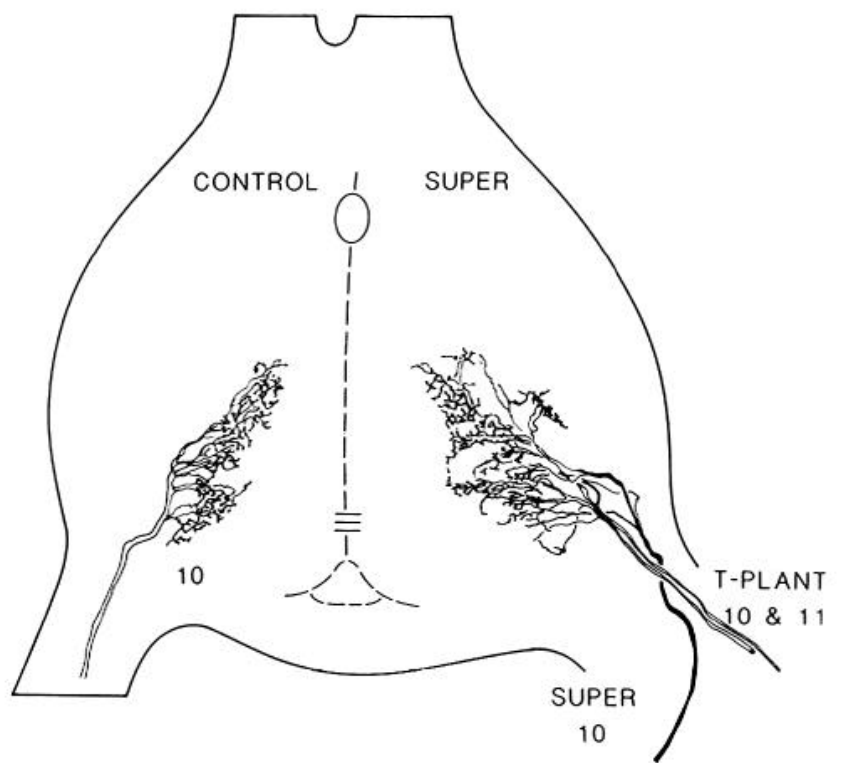

Figure 7. The overlap of arbors from transplanted and supernumerary neurons projecting into a single glomerulus. In this preparation transplanted neurons 10 and 11 were stained (two neurons to ensure success) and, simultaneously, supernumerary neuron 10 was stained.
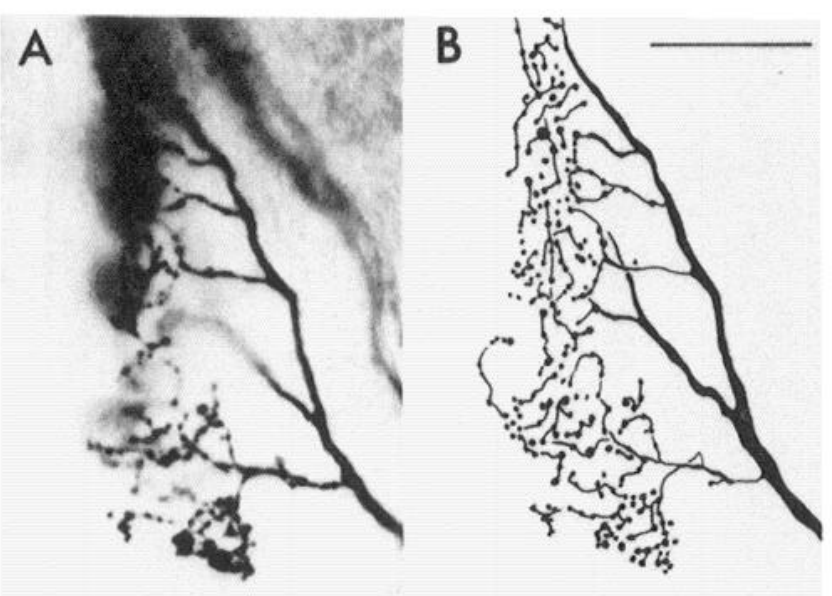

Figure 8. Varicosities within the terminal arborization of neuron $60 . A$, Photomicrograph of a small region of the terminal arborization to illustrate the original material. $B$, The stylized drawing used to obtain the number of varicosities within the arbor shown in $A$. Each dot in the drawing, independent of size, was counted as a varicosity and the results were tabulated in Table II. Calibration bar, $40 \mu \mathrm{m}$.
TABLE II

Quantitative comparison of terminal arbors

\begin{tabular}{lcc}
\hline \multirow{2}{*}{ Preparation } & \multicolumn{2}{c}{ Number of Varicosities } \\
\cline { 2 - 3 } $3-11-82-3$ & 161 & Control \\
$1-6-82-2$ & 286 & 234 \\
$3-24-82-7$ & 268 & $-{ }^{a}$ \\
$4-12-82-2$ & 200 & 250 \\
$4-8-82-5$ & 188 & 207 \\
$3-11-82-1$ & 247 & - \\
$1-11-82-1$ & 148 & - \\
$3-3-82-15$ & - & - \\
& $\overline{\mathrm{x}}=214 \pm 54$ & 199 \\
& Supernumerary & Control \\
\hline $9-16-81-6$ & 220 & 255 \\
$4-8-82-23$ & 191 & - \\
$4-8-82-17$ & 214 & 213 \\
$3-11-82-5$ & 137 & - \\
$9-21-81-5$ & 193 & 232 \\
& $\overline{\mathrm{x}}=191 \pm 33$ & \\
\hline & Transplant & Control \\
\hline $3-29-82-11$ & 214 & 221 \\
$1-26-82-11$ & 236 & 221 \\
$3-29-82-18$ & 277 & - \\
$4-8-82-24$ & 226 & $\overline{\mathrm{x}}=226 \pm 19$ \\
& $\overline{\mathrm{x}}=238 \pm 27$ & \\
\hline
\end{tabular}

${ }^{a}-$, none.

would be different if the duplicate projections in the supernumerary case produced some type of competitive interaction. However, there were no significant differences between these two groups, nor were there significant differences in any other pair-wise comparison of the data in Table II. Thus it appears that duplicate projections do not affect each others' growth by arborizing in the same area. This lack of interaction and the lack of cercus-specific domains suggest that competitive interactions are not involved in the growth of these cercal afferent projections.

Variations in axon trajectory and terminal arborizations. Neurons 40 and 60, although they are situated close to one another on the normal cercus and their terminal arbors overlap in the CNS, have widely divergent axon trajectories. The overlap in terminal arborizations reflects their adjacent positions on the cercus but their very different axon trajectories suggest that there are equivalent ways to reach a given target region. Control neurons exhibit alternative trajectories in a very small number of cases $(\leq 5 \%)$. However, experimental neurons exhibited axon trajectory errors much more often (Table III). For example, supernumerary neuron 40 exhibited a ventromedial trajectory (e.g., Fig. $2 D$ ) in $46 \%$ of the cases and neuron 60 had processes in the dorsolateral tract $32 \%$ of the time.

One possible cause for the axon trajectory alterations was a simple mechanical one. The location of the supernumerary structure might deflect the axons of supernumerary neurons into unusual tracts. However, neurons on supernumerary cerci at particular locations showed no obvious preference for one or the other axon trajectories, eliminating this possibility. 
Regeneration of a cercus. The altered axon trajectories of neurons 40 and 60 as well as other subtle differences between the terminal arbors of supernumerary neurons and their pristine homologs on the control cercus led us to examine the regeneration process itself as a cause for altered axon trajectories. It is well known that if a cercus is amputated early in postembryonic life, it will regenerate and function relatively normally (Palka and Edwards, 1974; Murphey et al., 1976). We therefore amputated cerci and allowed them to regenerate with a time course similar to that for the generation of supernumerary cerci and then examined the arborization of sensory neurons. Neurons on the regenerate were in general indistinguishable from their homologs on the control cercus (e.g., neuron 10, Fig. $3 B$ ). However, neurons 40 and 60 on these regenerate cerci again exhibited altered axon trajectories in proportions similar to those seen for supernumerary neurons (Table III). Forty percent of regenerate neuron 40 s selected the ventromedial tract rather than the normal dorsolateral tract, and $31 \%$ of neuron 60 s had branches in the dorsolateral tract (Table III). It thus appears that the regeneration process itself leads to errors in axon trajectory.

In spite of these errors in axon trajectory and the slight shifting of arborizations, the locations of the arbors are those normally seen. Further, the complexity of the arbor, as measured by the number of varicosities in the terminal, was not altered by the axon trajectory errors (Table II). Equally important, these neurons were never seen to invade neuropil characteristic of other neurons such as 80 or 90 .

TABLE III

Altered axon trajectories

\begin{tabular}{|c|c|c|c|}
\hline \multirow[b]{2}{*}{ Type of Neuron } & \multicolumn{2}{|c|}{ Axon Trajectory } & \multirow[b]{2}{*}{ Other } \\
\hline & $\begin{array}{c}\text { Dorsolateral or Bifurcate } \\
\text { (Terminal arbor dorsolateral } \\
\text { and ventromedial) }\end{array}$ & $\begin{array}{l}\text { Ventral (Termi- } \\
\text { nal arbor ven- } \\
\text { tromedial only) }\end{array}$ & \\
\hline \multicolumn{4}{|l|}{$\begin{array}{l}\text { Supernumer- } \\
\text { ary }\end{array}$} \\
\hline $\begin{array}{l}\text { Neuron } 40 \\
(N=11)\end{array}$ & $5(46 \%)^{a}$ & $5(46 \%)^{b}$ & $1(9 \%)$ \\
\hline $\begin{array}{l}\text { Neuron } 60 \\
(N=19)\end{array}$ & $6(32 \%)$ (2 bifurcate) & $12(63 \%)$ & $1(5 \%)$ \\
\hline \multicolumn{4}{|l|}{$\begin{array}{l}\text { Left } \rightarrow \text { right } \\
\text { transplant }\end{array}$} \\
\hline $\begin{array}{l}\text { Neuron } 40 \\
\quad(N=23)\end{array}$ & $17(74 \%)$ & $6(26 \%)$ & $-d$ \\
\hline $\begin{array}{l}\text { Neuron } 60 \\
\quad(N=38)\end{array}$ & $12(32 \%)$ ( 1 bifurcate $)^{c}$ & $25(66 \%)$ & $1(2 \%)$ \\
\hline \multicolumn{4}{|l|}{ Regenerate } \\
\hline $\begin{array}{l}\text { Neuron } 40 \\
(N=10)\end{array}$ & $4(40 \%)$ & $4(40 \%)$ & $1(7 \%)$ \\
\hline $\begin{array}{l}\text { Neuron } 60 \\
\quad(N=11)\end{array}$ & $6(43 \%)$ (4 bifurcate) & $7(50 \%)$ & $7 \%$ \\
\hline \multicolumn{4}{|l|}{ Control } \\
\hline $\begin{array}{l}\text { Neuron } 40 \\
(N=57)\end{array}$ & $55(96 \%)$ & 0 & $2(4 \%)$ \\
\hline $\begin{array}{l}\text { Neuron } 60 \\
\quad(N=82)\end{array}$ & $4(5 \%)$ & $76(93 \%)$ & $2(2 \%)$ \\
\hline
\end{tabular}

${ }^{a}$ See Figure 6, transplant neuron 40 for an example of this type.

${ }^{b}$ See Figure 2, supernumerary neuron 40 for ân example of this type.

${ }^{c}$ See Figure 6, transplant neuron 60 for an example of this type.

${ }^{d}$-, none.

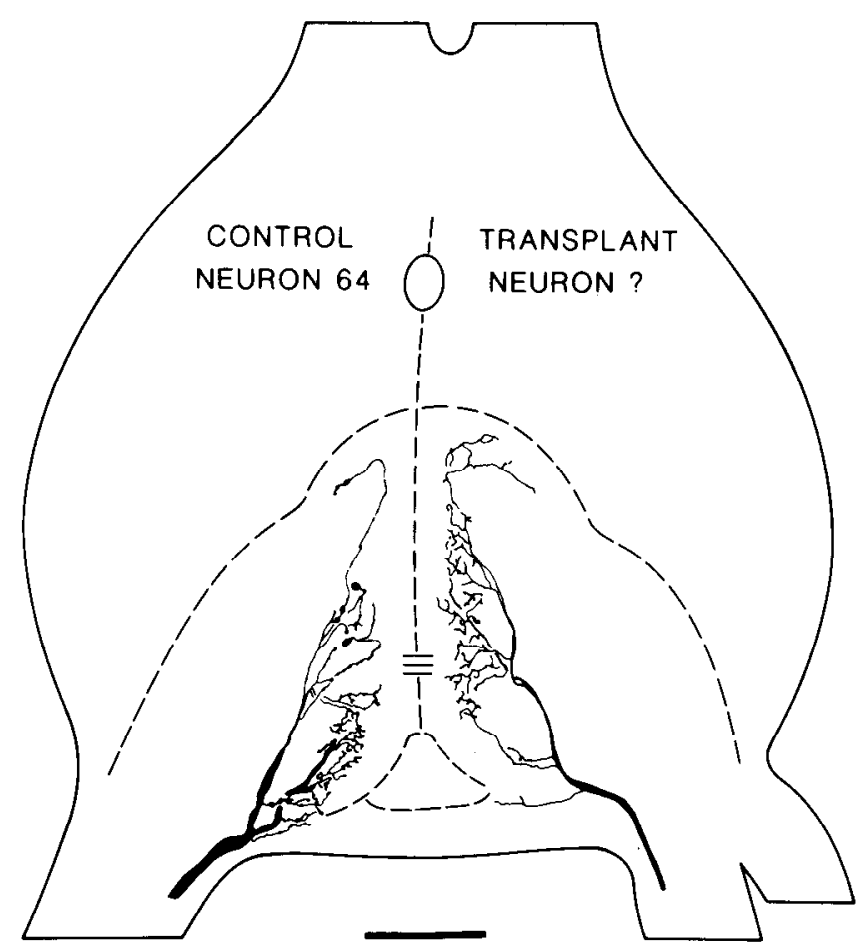

Figure 9. Predicting a neuron's target area. The cell on the right innervated an unidentifiable receptor near the margin of the transplant and supernumerary cerci. The cell on the left, neuron 64, was selected to serve as an estimate of what the experimental neuron should look like based on its location in the regenerated tissue (see the text for details). Calibration bar, $100 \mu \mathrm{m}$.

Neurons near graft margins. Near the base of the cercus where the supernumerary and transplant merge, there are clavate reccptors of unknown identity. One can often find such unidentified receptors between receptor rows which are clearly identified as belonging to the supernumerary and transplant cercus, respectively. We used these identified rows as landmarks to interpolate positions likely to be between the landmarks. We then stained an identified receptor on the control cercus which we thought would represent an equivalent location. Most experimental neurons projected just as we predicted, based on their position in the intercalary tissue (Fig. 9). In the example shown, we predicted that an unidentified receptor would project in a manner similar to that of cells in the neuron 60 row. We therefore stained the unidentified neuron and, since it was proximal, stained cell 64 on the control cercus. Clearly the experimental neuron projected to the region of neuropil characteristic of neurons in row 60 . It is worth pointing out that the experiment is of necessity done blind because we can only determine the result of staining the two neurons after making the prediction. The results support the idea that location of the cell body in the periphery in some manner influences the location of its terminal arbor in the CNS.

Establishment of functional connections between supernumerary neurons and an appropriate central target. The anatomical results presented thus far provide strong evidence that supernumerary neurons project to target areas consistent with their position of the super- 


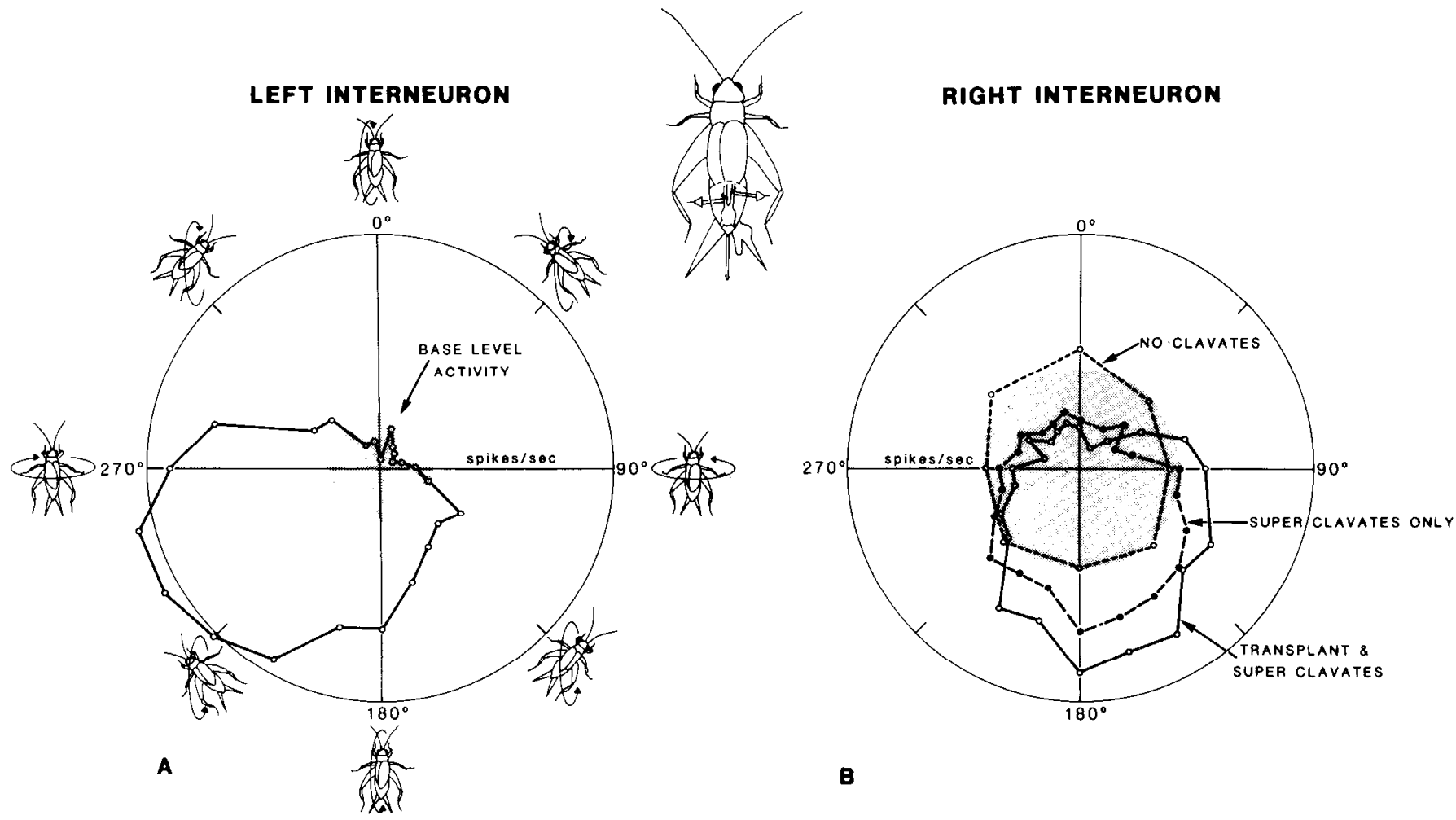

Figure 10. Establishment of functional connections between supernumerary neurons and an appropriate central target. Polar plots of receptive fields for the position-sensitive interneurons were obtained by tilting the animal $60^{\circ}$ at $15^{\circ}$ increments around the yaw axis. The stippled region at the center of each receptive field represents the base level activity recorded when the animal was at primary orientation. Points plotted within this region represent a decrease in action potential frequency and points plotted outside of the stippled area represent an increase in the action potential frequency as a result of displacement. Each datum point is the mean for six trials. $A$, Receptive field from the control left interneuron. $B$, Receptive fields from the experimental right interneuron. In this animal a left cercus was transplanted into the host right socket, resulting in the generation of medial and lateral supernumerary cerci (see inset diagram of the cricket). Initially, all clavate hairs were free to move on the transplanted and supernumerary cerci ( solid line). Next, clavate hairs on the transplanted cercus were selectively deleted by plucking, leaving only clavate hairs on the medial supernumerary cercus free to move (bold dashed line, solid circles). Finally, the remaining clavate hairs were deleted (dashed line, open circles).

numerary cercus. The following experiments were performed to determine whether functional connections were established between supernumerary neurons and the target neuron.

The clavate receptors provide the primary afferent input to a pair of bilaterally symmetric interneurons located in the CNS (Sakaguchi and Murphey, 1981). This clavate sensory system is thought to apprise the animal of equilibrium position information (Bischof, 1975). Receptive fields for the position-sensitive interneurons (PSI) have been determined by tilting the animal on a goniometer stage which resulted in modulation of the action potential frequency recorded from the PSI. When plotted in polar coordinates, control receptive fields for each PSI predominantly occupy one quadrant. The left positional intcrncuron (its axon is in the left connective) has its receptive field in the left posterior quadrant and has an axis of maximal sensitivity when the specimen is tilted to the left rear (i.e., $225^{\circ}$ ) (Fig. 10). The receptive field for the right interneuron is a mirror image occupying the right posterior quadrant.

We examined the central connections of clavate sensory neurons on both the transplanted and supernumerary cerci. In all experiments the left neuron served as an internal control for the experimental, right neuron. Re- cordings from experimental animals revealed that connections were established with the appropriate interneuron ipsilateral to the supernumerary cercus (Fig. 10B). The position, with respect to body axes, of the transplant and supernumerary cerci are distorted by their respective partners. For example, the medial supernumerary is often pointing directly posterior, while a normal right cercus points to the right rear, and the orientation of the receptive field reflects this. Figure 10 illustrates the results from an experiment in which we successively deleted clavate hairs to examine the ability of clavate receptors located on a supernumerary cercus to excite the appropriate positional interneuron. With all clavate hairs free to move on the experimental cerci, a somewhat normal receptive field was obtained for the right positional interneuron (Fig. 10B). When the clavate hairs on the transplanted cercus were removed, the interneuron was still activated by displacement, demonstrating that the clavate receptors on the supernumerary cercus were capable of driving the host positional interneuron (Fig. $10 B$, Super clavates only). When the clavate receptors on the supernumerary cercus were finally deleted, the right positional interneuron no longer responded to positional changes (Fig. 10B, No clavates).

Thus it is clear that the clavate sensory neurons on 
supernumerary cerci do form functional connections with appropriate central targets and they do so in a normal manner. In all animals tested, such connections were established between supernumerary clavate sensory neurons and the ipsilateral positional interneuron (Fig. 10).

\section{Discussion}

The finding that supernumerary sensory neurons project to the CNS in a manner consistent with their location on the supernumerary cercus has a number of implications for hypotheses which would explain pattern formation in the CNS. There are a number of cues which might be responsible for the assembly of somatotopic afferent projections. They can be assigned to at least four basic groups. For simplicity we will call them mechanical cues, spatiotemporal cues, competitive interactions, and positional cues. Many of the models for the assembly of somatotopic connections utilize multiple cues and the actual mechanism in any given case may well lie in some combination of these posibilities. The results presented here bear directly on the importance of these cues in assembling the cercal afferent projection.

Mechanical cues. One simple mechanism for creating an organized projection would be to arrange the mechanics of growth and the location of cells such that the axons of cells are automatically directed into appropriate areas of the CNS (e.g., see Harrison, 1910; Weiss and Taylor, 1944; Macagno, 1979; Scholes, 1979; Bodick and Levinthal, 1980). Such a mechanism might be expected to exist for the cercal afferent system since location in the periphery, in the nerve, and ultimately in the ganglion are fairly simply related (see Murphey, 1981 for details). However, many axons from supernumerary structures take courses quite independent of their homologs on the transplant, often arriving at the CNS by a separate nerve. The axons of the two supernumerary and transplant nerves are then "organized" at their intersection near the ganglion, all arriving at the appropriate target areas through an interdigitation of the two bundles of axons (Fig. 11). Some supernumerary neurons cross all of their neighbors at the nerve-ganglion junction to arrive at their normal destination. Thus, near the ganglion of these experimental animals the cercal nerve exhibits a reorganization reminiscent of the optic nerve of cichlid fish as it approaches the tectum (Scholes, 1979), a reorganization which is difficult to explain by simple mechanics. Furthermore, when we examined neurons whose axon trajectories were altered by transplantation (Murphey et al., 1981), regeneration, or supernumerary production (Table III), very few altered axon trajectory errors were correlated with altered terminal arborizations; most neurons arrive at their proper target regions even with incorrect trajectories. Finally, it is now clear that many neurons at polar opposite locations on the circumference of the normal cercus can have very different axon trajectories but identical target regions in the ganglion (Bacon and Murphey, 1981). All of this leads us to conclude that in the cercal system, simple mechanics must play a minor role in controlling the axon trajectory, and more important, that axon trajectory is not tightly coupled to the location of the terminal arborization of these cricket sensory neurons.

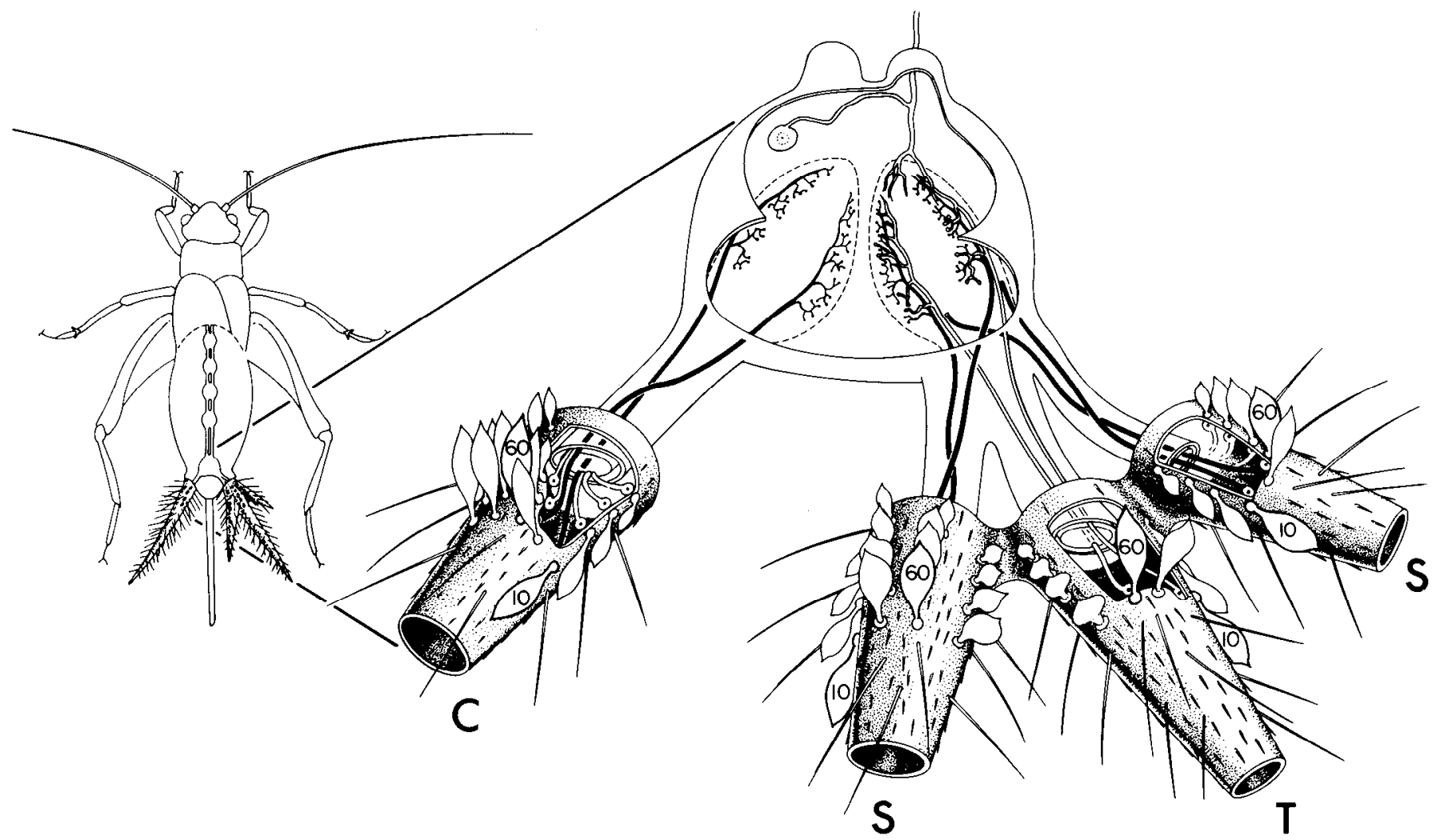

Figure 11. Artist's summary of the major results. The control cercus and projections of control neurons 10 and 60 are shown on the left. The experimental cercus and neurons are shown on the right. Note that transplanted and supernumerary neurons arborized normally and that the homologous neurons arborize on the same area. $C$, control cercus; $T$, transplanted cercus (note it is left handed); $S$, supernumerary cerci (note they are right handed). 
Temporal cues. The birthday of a clavate sensory neuron on a normal cercus is correlated with the location of its arbor in the CNS (Murphey et al., 1980). Thus it was possible that the time of arrival in the CNS might be used to order the terminal arborizations in the proximodistal axis. Supernumerary neurons provide information relevant to this point because they have a much later birthday than their homologs on the transplanted cercus. The earliest supernumerary receptors appear 19 days postoperative, and we know that the growth cones of the transplanted neurons reach the CNS at least as early as 10 days postoperative (W. W. Walthall and R. K. Murphey, manuscript in preparation). 'Thus, there is at least a 9-day separation in the time of arrival of the growth cones of homologous supernumerary and transplant neurons in the CNS. Since the neurons are produced during the intermolt period just prior to their appearance on the cercus, this estimate would have to be reduced to a 5- or 6-day difference. However, this is the worst-case scenario. Most supernumerary limbs take longer to mature and the difference in time of arrival is magnified for more proximal neurons, such as neuron 15 , which is present on the transplant at the time of surgery but does not appear on the supernumerary until more than 19 days postoperative. Since we have shown that homologous cells arborize in identical regions of the target in spite of different birthdays and different times of arrival in the target area, birth order cannot be a critical variable in setting up this axis of the afferent projection. This is consistent with numerous other experiments in which we have disrupted the birth order/target area correlation (Murphey et al., 1981), and we conclude that time of arrival at the CNS is not a relevant variable in assembling the cercal afferent projection.

Competitive interactions. Implicit in the time of arrival hypothesis just discussed is the idea that early arriving cells occupy certain synaptic sites and exclude late arrivals from their domain by some competitive interaction. As presently defined (Purves, 1980), competitive interactions have not been observed in insects, and the present experiments might have been expected to reveal such interactions if they exist in the cercal system. For example, neurons with homologous positions on supernumerary and transplant cerci might have excluded one another or divided the target region into separate domains as happens with supernumerary eyes in frogs (ConstantinePaton and Law, 1978). In fact, the homologous neurons interdigitate and supernumerary and transplant do not set up separate, cercus-specific projections (Fig. 11). In addition, a quantitative analysis of the terminal arbors of the various neurons failed to reveal any differences between the supernumerary neurons and appropriate controls (Table II). Thus we are unable to demonstrate competitive interactions among afferents in the cercal sensory system. Based on the information available, we conclude that competitive interactions between afferents do not exist in the cercal sensory system. (Results which have appeared in abstract form and which we called "competitive" (Walthall and Murphey, 1981) are presently being reassessed and appear to be due to other mechanisms (W. W. Walthall and R. K. Murphey; manuscript in preparation.)) Similar analyses in Drosophila, where the number of receptors was altered genetically, point to a similar conclusion (Ghysen, 1980; Burt and Palka, 1982). Thus, in crickets and flies, there is as yet no evidence for competitive interactions. These studies focus attention on the possibility that such interactions do not exist in the insect CNS, a possibility which must be carefully tested.

Positional influences. There is a clear correlation between the position of a neuron's soma and the location of its arborization in the CNS. Classically, in the literature on insect epidermis, the evidence for positional influences in differentiation has depended on the regeneration of areas of tissue at graft boundaries between normally disparate areas of the body. Such regeneration, called intercalary regeneration, which includes the production of supernumerary limbs, supports the positional information model because structures are formed de novo and the nature of the structure is predictable and consistent with position, with respect to the graft boundaries, in the regenerate tissue (Wolpert, 1969; French et al., 1976; Bate, 1978; Palka, 1980). Since the sensory arrays on supernumerary structures are quite normal in structure and orientation and their symmetry is consistent with the model of French et al. (1976), it seems reasonable to conclude that cerci must be subject to the same rules for regeneration as other insect limbs and imaginal discs (French et al., 1976). More to the point, the areas of the CNS invaded by the supernumerary cercal neurons are exactly lhose that would be predicted based on their position on the supernumerary cerci. This suggests that the rules which lead to pattern formation in the epidermis are in some way translated to rules for the projection of sensory neurons. Further support for this idea was provided by the examination of neurons near graft boundaries. In these cases the identity of the neuron was unknown and we estimated its identity by determining its position with respect to nearby markers. This interpolated position was an accurate indicator of its arbor position in the CNS. In summary, the most economical hypothesis to explain the results presented here, as well as a host of other experiments (Bate, 1973; Ghysen, 1980, Murphey et al., 1981), is that one step in the differentiation of cercal sensory neurons is position dependent and that this step determines the choice of a target region. While we have phrased our experiments in the context of developmental work on the insect epidermis and the "positional information" model commonly used there, this model is similar to Sperry's (1963) chemoaffinity model.

\section{References}

Bacon, J. P., and R. K. Murphey (1981) Cricket cercal filiform hairs have a somatotopic projection in the terminal ganglion. Soc. Neurosci. Abstr. 7: 4.

Bate, C. M. (1973) The mechanism of the pupal gin trap. I. Segmental gradients and the triggering sensilla. J. Exp. Biol. 59: $95-107$.

Bate, C. M. (1976) Embryogenesis of an insect nervous system. I. A map of the thoracic and abdominal neuroblasts in Locusta migratoria. J. Embryol. Exp. Morphol. 35: 107-123.

Bate, C. M. (1978) Development of sensory systems in arthropods. In Handbook of Sensory Physiology, M. Jacobson, ed., Vol. 9; pp. 2-53, Springer-Verlag; New York.

Bischof, H. J. (1975) Die keulenformigen sensillen auf den Cerci 
der Grille Gryllus bimaculatus als Schwerereceptoren. J. Comp. Physiol. 98: 277-288.

Bodick, N., and C. Levinthal (1980) Growing optic nerve fibers follow nieghbors during embryogenesis. Proc. Natl. Acad. Sci. U. S. A. 77: 4374-4378.

Burt, R., and J. Palka (1982) The central projections of mesothoracic sensory neurons in wildtype Drosophila and $B i$. thorax mutants. Dev. Biol. 90: 99-109.

Constantine-Paton, M., and M. I. Law (1978) Eye-specific termination bands in tecta of three-eyed frogs. Science 202: 639641.

Edwards, J. S., and J Palka (1974) The cerci and abdominal giant fibers of the house cricket Acheta domesticus. I. Anatomy and physiology of normal adults. Proc. R. Soc. Lond. (Biol.) 185: 83-103.

French, V. I., P. J. Bryant, and S. V. Bryant (1976) Pattern regulation in epimorphic fields. Science 193: 969-981.

Ghysen, A. (1980) The projection of sensory neurons in the central nervous system of Drosophila: Choice of the appropriate pathway. Dev. Biol. 78: 521-541.

Harrison, R. G. (1910) The outgrowth of nerve fibers as a mode of protoplasmic movement. J. Exp. Zool. 9: 787-846.

Lawrence, P. A. (1966) Development and determination of hairs and bristles in the milkweek bug Oucopeltus fasciatus. J. Cell Sci. 1: 475-498.

Macagno, E. R. (1979) Cellular interactions and pattern formation in the development of the visual system of Daphnia magna (Crustacea, Branchiopoda). I. Interactions between embryonic reticular fibers and laminar neurons. Dev. Biol. 73: 206-238.

Murphey, R. K. (1981) The structure and development of a somatotopic map in crickets: The cercal afferent projection. Dev. Biol. 88: 236-246.

Murphey, R. K., S. G. Matsumoto, and B. Mendenhall (1976) Recovery from deafferentation by cricket interneurons after reinnervation by their peripheral field. J. Comp. Neurol. 169: $335-346$.

Murphey, R. K., A. Jacklet, and L. Schuster (1980) A topographic map of sensory cell terminal arborizations in the cricket CNS: Correlation with birthday and position in a sensory array. J. Comp. Neurol. 191: 53-64.
Murphey, R. K., S. E. Johnson, and W. W. Walthall (1981) The effects of transplantation and regeneration of sensory neuron on a somatotopic map in the cricket central nervous system. Dev. Biol. 88: 247-258.

Murray, M., S. Sharma, and M. A. Edwards (1982) Target regulation of synaptic number in the compressed retinotectal projection of goldfish. J. Comp. Neurol., in press.

Palka, J. (1980) Theories of pattern formation in insect neural development. Adv. Insect Physiol. 14: 256-349.

Palka, J., and J. S. Edwards (1974) The cerci and abdominal giant fibres of the house cricket, Acheta domesticus. II. Regeneration and effects of chronic deprivation. Proc. R. Soc. Lond. (Biol.) 185: 105-121.

Palka, J., and M. Schubiger (1975) Central connections of receptors on rotated and exchanged cerci of crickets. Proc. Natl. Acad. Sci. U. S. A. 72: 966-969.

Purves, D. (1980) Neuronal competition. Nature 287: 585-586.

Sakaguchi, D. S., and R. K. Murphey (1981) Synaptic interactions between the clavate sensory neurons of crickets and an identified interneuron. Soc. Neurosci. Abstr. 7: 4.

Scholes, J. H. (1979) Nerve fibre topography in the retinal projection to the tectum. Nature 278: 620-624.

Shankland, M. (1981) Development of a sensory afferent projection in the grasshopper embryo. I. Growth of peripheral pioneer axons within the central nervous system. J. Embryol. Exp. Morphol. 64: 169-185.

Sperry, R. W. (1963) Chemoaffinity in the orderly growth of nerve fiber patterns and connections. Proc. Natl. Acad. Sci. U. S. A. 50: $703-710$.

Strausfeld, N. J. (1971) The organization of the insect visual system. I. Projections and arrangements of neurons in the lamina ganglionaris of Diptera. Z. Zellforsch. 121: 377-441.

Walthall, W. W., and R. K. Murphey (1981) Competitive interactions and the formation of a somatotopic map in an insect. Soc. Neurosci. Abstr. 7: 4 .

Weiss, P., and A. C. Taylor (1944) Further experimental evidence against neurotropism in nerve regeneration. J. Exp. Zool. 95: 233-254.

Wolpert, T. (1969) Positional information and the spatial pattern of cellular differentiation. J. Theor. Biol. 25: 1-47. 